\title{
A Bayesian cost-effectiveness analysis of a telemedicine-based strategy for the management of sleep apnoea: a multicentre randomised controlled trial
}

\author{
Valentina Isetta, ${ }^{1,2,3}$ Miguel A Negrín, ${ }^{4}$ Carmen Monasterio, ${ }^{3,5}$ Juan F Masa, ${ }_{1}^{3,6}$ \\ Nuria Feu, ${ }^{7}$ Ainhoa Álvarez, ${ }_{1}^{8}$ Francisco Campos-Rodriguez, ${ }_{1}^{9}$ Concepción Ruiz, ${ }^{10}$ \\ Jorge Abad, ${ }^{3,11}$ Francisco J Vázquez-Polo, ${ }^{4}$ Ramon Farré, ${ }^{1,2,3}$ Marina Galdeano, ${ }^{12}$ \\ Patricia Lloberes, ${ }^{3,13}$ Cristina Embid, ${ }^{3,10}$ Mónica de la Peña, ${ }^{3,14}$ Javier Puertas, ${ }^{15,16}$ \\ Mireia Dalmases, ${ }^{3,10}$ Neus Salord, ${ }^{3,5}$ Jaime Corral, ${ }^{3,6}$ Bernabé Jurado, ${ }^{7}$ \\ Carmen León, ${ }_{1}^{10}$ Carlos Egea, ${ }^{8}$ Aida Muñoz, ${ }^{11}$ Olga Parra, ${ }^{3,12}$ Roser Cambrodi, ${ }^{13}$ \\ María Martel-Escobar, ${ }^{4}$ Meritxell Arqué, ${ }^{14}$ Josep M Montserrat, ${ }^{3,10}$ the SPANISH \\ SLEEP NETWORK
}

\begin{abstract}
- Additional material is published online only. To view please visit the journal online (http://dx.doi.org/10.1136/ thoraxjn-2015-207032).

For numbered affiliations see end of article.
\end{abstract}

\section{Correspondence to} Dr Valentina Isetta, Unitat de Biofísica i Bioenginyeria, Facultat de Medicina, Universitat de Barcelona, Casanova 143, Barcelona, 08036, Spain valentina.isetta@ub.edu

Received 11 March 2015 Revised 2 July 2015 Accepted 28 July 2015 Published Online First 26 August 2015

\section{CrossMark}

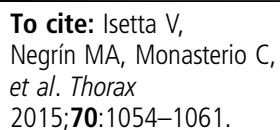

\begin{abstract}
Background Compliance with continuous positive airway pressure (CPAP) therapy is essential in patients with obstructive sleep apnoea (OSA), but adequate control is not always possible. This is clinically important because CPAP can reverse the morbidity and mortality associated with OSA. Telemedicine, with support provided via a web platform and video conferences, could represent a cost-effective alternative to standard care management.
\end{abstract}

Aim To assess the telemedicine impact on treatment compliance, cost-effectiveness and improvement in quality of life (QoL) when compared with traditional face-to-face follow-up.

Methods A randomised controlled trial was performed to compare a telemedicine-based CPAP follow-up strategy with standard face-to-face management. Consecutive OSA patients requiring CPAP treatment, with sufficient internet skills and who agreed to participate, were enrolled. They were followed-up at 1, 3 and 6 months and answered surveys about sleep, CPAP side effects and lifestyle. We compared CPAP compliance, costeffectiveness and QoL between the beginning and the end of the study. A Bayesian cost-effectiveness analysis with non-informative priors was performed.

Results We randomised 139 patients. At 6 months, we found similar levels of CPAP compliance, and improved daytime sleepiness, QoL, side effects and degree of satisfaction in both groups. Despite requiring more visits, the telemedicine group was more cost-effective: costs were lower and differences in effectiveness were not relevant. Conclusions A telemedicine-based strategy for the follow-up of CPAP treatment in patients with OSA was as effective as standard hospital-based care in terms of CPAP compliance and symptom improvement, with comparable side effects and satisfaction rates. The telemedicine-based strategy had lower total costs due to savings on transport and less lost productivity (indirect costs).

Trial register number NCT01716676.

\section{Key messages}

What is the key question?

- What is the clinical and economic impact of a telemedicine-based approach for the follow-up of continuous positive airway pressure (CPAP) treatment in patients with obstructive sleep apnoea (OSA)?

What is the bottom line?

- Adherence to CPAP treatment is essential for treatment success; telemedicine could provide a cost-effective alternative to the standard face-to-face approach of CPAP follow-up.

\section{Why read on?}

- This multicentre randomised controlled trial provides evidence that a telemedicine-based strategy for the CPAP follow-up of OSA patients is as effective as the face-to-face approach but has lower cost for society.

\section{INTRODUCTION}

Obstructive sleep apnoea (OSA) is a common condition that causes significant morbidity and mortality and increased use of healthcare resources. ${ }^{1} 2$ Currently, improved case detection and the resulting higher healthcare demands have not been accompanied by any real improvement in OSA management. In addition, health resources assigned to OSA and its treatment have been found to be inadequate. $^{3}$ Continuous positive airway pressure (CPAP) is the optimal treatment for OSA. Nevertheless, many patients fail to use CPAP adequately; this is clinically relevant since increased adherence reduces symptoms and comorbidities. ${ }^{4}$ Optimising adherence is therefore an essential 
aspect of patient management and different educational and technical measures have been proposed. ${ }^{5}$ 6-10 However, adequate provision of such support is not always possible, and overloaded sleep centres can be hard-pressed, especially after recent healthcare budget cuts. Therefore, alternative and costeffective approaches are urgently needed to improve OSA management and to promote CPAP compliance. A possible approach is telemedicine, defined here as the use of information and communication technology (ICT) to provide clinical care for a patient at a distance. ${ }^{11}$ However, conflicting results have been found $^{12} 13$ and the economic impact of telemedicine on OSA management is still unclear. ${ }^{14-18}$ We conducted a multicentre randomised controlled trial (RCT) to evaluate the efficacy of a new telemedicine-based strategy for OSA patients under CPAP treatment. Specifically, we assessed its impact on treatment compliance, sleepiness, quality of life (QoL) and cost-effectiveness when compared with traditional face-to-face follow-up.

\section{METHODS}

\section{Study design}

This RCT was approved by the ethics committees of the eight participating hospitals in Spain. All patients provided informed consent prior to participation. Patients were randomly assigned to either standard face-to-face follow-up (control group) or to telemedicine-based follow-up for 6 months (figure 1).

\section{Population}

Participants were prospectively enrolled between December 2011 and December 2013. In accordance with Spanish health service guidelines, all enrolled patients were classified as requiring CPAP treatment after an overnight study. ${ }^{19}$ Exclusion criteria were as follows: severe sleepiness, severe nasal obstruction, pregnancy, psychiatric disease, dangerous employment, clinical instability and current or previous treatment for OSA. We excluded patients who lacked sufficient internet skills or refused to participate in the study. Both groups were equipped with conventional masks and received the same instructions regarding initial CPAP use.

\section{Procedures}

Clinical evaluation and follow-up

Patients' baseline characteristics were collected at the initial visit. In addition, EuroQol-5D, the Epworth Sleepiness Scale (ESS), the Functional Outcomes of Sleep Questionnaire (FOSQ) and the Quebec Sleep Questionnaire (QSQ) were administered. At 6 months, data on CPAP compliance, satisfaction and side effects were gathered and questionnaires repeated. Data related to costs were collected for the entire process. At intermediate visits (months 1 and 3), additional information was recorded regarding side effects, lifestyle changes and actions taken.

\section{Sleep studies}

All patients underwent a sleep study which was scored manually by trained personnel. ${ }^{19}$ Optimal CPAP pressure was titrated by an auto CPAP device to obtain a fixed pressure. ${ }^{20}$ Tests were repeated if patients claimed to sleep less than $4 \mathrm{~h}$, or when less than $5 \mathrm{~h}$ of recording were available.

\section{Randomisation}

Participants who completed the baseline visit were randomly assigned $(1: 1)$ to the control or telemedicine group. Randomisation was at an individual level without restriction
Figure 1 Study flowchart. The two groups received CPAP treatment follow-up via two different strategies: conventional follow-up, which consisted of face-to-face hospital visits (the control group), and telemedicine-based follow-up, using a website and televisits (the telemedicine group). The main outcomes were CPAP compliance, QoL and

cost-effectiveness. During the intermediate visits, only questionnaires were administered and some corrective actions were recommended if required. CPAP, continuous positive airway pressure; ITT, intention-to-treat; OSA, obstructive sleep apnoea; PP, per protocol; QALY, quality-adjusted life year; QoL, quality of life.

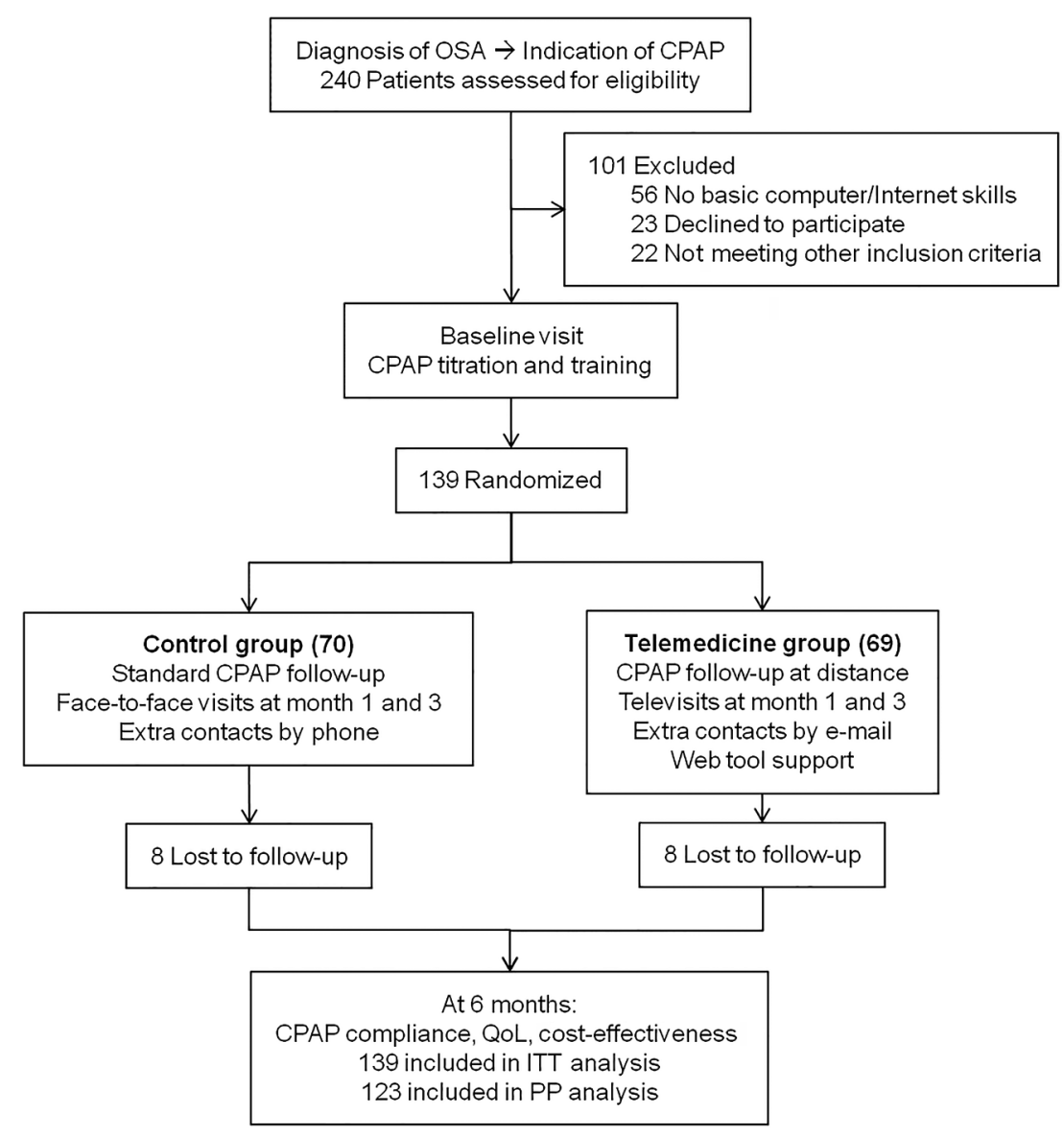


(ie, no blocking) and was completely automated by use of an unseen random number function embedded in the data collection website code. There was no clinician involvement at this stage, and the software only revealed the allocation group when an investigator provided the data of a fully eligible patient, thus guaranteeing concealment of the randomisation sequence.

\section{Telemedicine-based follow-up}

Patients randomised to the telemedicine group received their follow-up at home supported by a website developed for this study, where they could find information about OSA and CPAP therapy, and a biweekly six-item questionnaire about their status, physical activity, sleep time, CPAP use and treatment side effects. Each centre's staff monitored questionnaire answers and communicated with patients through the website messaging tool to solve treatment-related problems. To participate, patients only required an internet-connected device with a microphone and webcam. Televisits via video conference were undertaken at months 1 and 3. We used Skype due to its availability, ease of use and good performance. ${ }^{21}$ Patients automatically received a confirmation email indicating the date and time of their appointment. Extra televisits or hospital visits were scheduled as necessary.

\section{Hospital-based follow-up}

As shown in figure 1, patients randomised to the control group had the same follow-up schedule as the telemedicine group, but attended the hospital. Specifically, they received standard face-to-face follow-up with visits at months 1, 3 and 6, and extra visits if needed.

\section{Outcomes}

The primary outcomes were: (1) CPAP compliance; and (2) the cost-effectiveness of the two follow-up strategies, compared using quality-adjusted life years (QALYs) as a measure of effectiveness. QALYs were estimated from the EQ-5D tariffs. ${ }^{22-24}$ Secondary outcomes included questionnaire changes from baseline, CPAP treatment side effects and patient satisfaction.

\section{Cost analysis}

We adopted a societal perspective in the cost-effectiveness analysis, where both direct and indirect costs were considered but immaterial costs were not taken into account. Direct health costs were associated with the use of healthcare resources, such as medical and nursing personnel salaries and all costs related to follow-up. These included material costs such as masks, humidifiers and medications used by patients, as well as the costs of extra visits to respiratory physicians, other specialists, general practitioners, nurses and emergency services. Two types of extra visits were defined: OSA-related and non-OSA-related. Unit costs were provided by the administrative departments of one of the participating hospitals.

Direct non-health costs referred to the travel expenses incurred by patients when attending hospital. Indirect costs referred to the opportunity costs of lost productivity and work due to follow-up visits; we considered time lost due to both hospital visits and video conferences. The final visit after 6 months was a face-to-face visit for both groups; this was included in the protocol to allow comparison with the data obtained at the first visit. However, in normal practice this face-to-face visit would not be needed for the telemedicine group. For this reason, the costs of transport to the 6-month visit were not considered in the cost analysis for the telemedicine group, and we assumed indirect costs similar to those for the intermediate visits.

The total costs were evaluated against the QALYs through a Bayesian cost-effectiveness analysis. Other complementary costeffectiveness analyses were assessed, taking into account changes in the ESS and CPAP treatment compliance.

\section{Statistical analysis}

We considered two analysis populations for this trial: an intention-to-treat (ITT) population (all randomised patients) and a per protocol (PP) population (all randomisation patients who finished the study). The planned sample size was based on the assumptions that $85 \%$ of the patients who were randomly assigned to treatment would meet the above definition of a PP sample for the non-inferiority test. The study was based on the assumption that the average compliance without intervention would be $4 \mathrm{~h}$ /day (SD 1.8). According to a one-sided type I error of 0.025 and $80 \%$ power to verify that CPAP compliance in the telemedicine group was not less than $1 \mathrm{~h} /$ day (SD 2), and assuming a $10 \%$ drop-out rate, the sample size was 60 patients in each group.

We reported means and SD for continuous variables with normal distribution and medians (1st quartile-3rd quartile) for those with non-normal distribution, and compared them using the $t$ test or the non-parametric Mann-Whitney test, respectively. Categorical variables are presented as number of patients (\%) and were compared using the $\chi^{2}$ test or Fisher exact test.

Conventional regression imputation techniques were used to estimate values for patients without valid CPAP use measurements at 6 months, assuming that missing data were missing at random. For the 16 patients with missing CPAP use at 6 months (eight in the control group and eight in the telemedicine group), we generated values based on sex, age, baseline snoring, witnessed apnoeas, choking episodes, nocturia, daytime sleepiness, non-restorative sleep, daytime fatigue, drowsiness during driving, nasal problems and ESS score, using stochastic regression imputations by a linear regression method.

Table 1 Baseline characteristics

\begin{tabular}{|c|c|c|c|}
\hline & $\begin{array}{l}\text { All patients } \\
(\mathrm{N}=139)\end{array}$ & $\begin{array}{l}\text { Telemedicine } \\
(\mathrm{N}=69)\end{array}$ & $\begin{array}{l}\text { Control } \\
(\mathrm{N}=70)\end{array}$ \\
\hline Men & $120(86 \%)$ & $59(85 \%)$ & $61(87 \%)$ \\
\hline Age (years) & $49.0(10.1)$ & $51.0(8.9)$ & $47.0(10.9)$ \\
\hline BMI $\left(\mathrm{kg} / \mathrm{m}^{2}\right)$ & $33.2(7.8)$ & $32.8(7.3)$ & $33.6(8.3)$ \\
\hline $\begin{array}{l}\text { Epworth Sleepiness } \\
\text { Scale }\end{array}$ & $10.7(4.7)$ & $10.5(4.6)$ & $10.8(4.8)$ \\
\hline Cardiovascular disease & $18(13 \%)$ & $11(16 \%)$ & $7(10 \%)$ \\
\hline Depression & $20(14 \%)$ & $13(19 \%)$ & $7(10 \%)$ \\
\hline Daytime fatigue & $97(70 \%)$ & $49(71 \%)$ & $48(69 \%)$ \\
\hline Drowsy driving & $73(52 \%)$ & $36(52 \%)$ & $37(53 \%)$ \\
\hline Nasal problems & $89(64 \%)$ & $46(67 \%)$ & $43(62 \%)$ \\
\hline $\begin{array}{l}\text { Apnea-Hypopnea Index } \\
\text { (event/h) }\end{array}$ & $49(35-46)$ & $45(35-70)$ & $52(35-62)$ \\
\hline $\begin{array}{l}\text { Oxygen Desaturation } \\
\text { Index }(\%)\end{array}$ & $46(27-67)$ & $43(27-67)$ & $48(27-67)$ \\
\hline СT90 (\%) & $12.0(3-31)$ & $13.0(3-30)$ & $11.5(3-31)$ \\
\hline $\begin{array}{l}\text { CPAP pressure } \\
\left(\mathrm{cm} \mathrm{H} \mathrm{H}_{2} \mathrm{O}\right)\end{array}$ & $9(8-11)$ & $9(8-11)$ & $9(8-10)$ \\
\hline
\end{tabular}

Intention-to-treat population.

Data are shown as mean (SD), median (1st quartile-3rd quartile) or number of patients (\%).

BMI, body mass index; CPAP continuous positive airway pressure. 
Table 2 Comparison of change from baseline in QoL, ESS and BMI between the telemedicine and control groups

\begin{tabular}{|c|c|c|c|c|c|c|c|c|}
\hline & \multicolumn{2}{|l|}{ Control } & \multicolumn{2}{|l|}{ Telemedicine } & \multirow{2}{*}{$\begin{array}{l}\text { LS mean* } t \\
\text { difference } \\
\text { (telemedicine } \\
\text { minus control) }\end{array}$} & \multicolumn{2}{|c|}{$\begin{array}{l}95 \% \mathrm{Cl} \text { for the } \\
\text { difference }\end{array}$} & \multirow[b]{2}{*}{ p Value } \\
\hline & $\begin{array}{l}\text { Baseline } \\
\text { Mean (SD) }(\mathrm{N}=70)\end{array}$ & $\begin{array}{l}\text { Follow-up } \\
\text { Mean (SD) (N=64) }\end{array}$ & $\begin{array}{l}\text { Baseline } \\
\text { Mean (SD) (N=69) }\end{array}$ & $\begin{array}{l}\text { Follow-up } \\
\text { Mean (SD) (N=64) }\end{array}$ & & Lower & Upper & \\
\hline \multicolumn{9}{|l|}{ EuroQol } \\
\hline EQ-5D & $0.84(0.18)$ & $0.88(0.20)$ & $0.78(0.23)$ & $0.82(0.19)$ & -0.03 & -0.09 & 0.03 & 0.38 \\
\hline EQ VAS & $6.43(2.07)$ & $7.36(1.88)$ & $6.74(1.69)$ & $6.80(2.18)$ & -0.48 & -1.10 & 0.15 & 0.13 \\
\hline \multicolumn{9}{|l|}{ Quebec } \\
\hline Hypersomnolence & $4.58(1.67)$ & $5.95(1.22)$ & $4.56(1.56)$ & $5.81(1.30)$ & -0.05 & -0.40 & 0.31 & 0.80 \\
\hline Diurnal symptoms & $4.72(1.57)$ & $5.92(1.12)$ & $4.35(1.60)$ & $5.68(1.40)$ & -0.05 & -0.41 & 0.32 & 0.81 \\
\hline Nocturnal symptoms & $4.34(1.47)$ & $5.80(1.34)$ & $4.38(1.29)$ & $5.50(1.33)$ & -0.20 & -0.61 & 0.21 & 0.33 \\
\hline Emotions & $5.01(1.48)$ & $5.91(1.39)$ & $4.69(1.41)$ & $5.61(1.38)$ & -0.07 & -0.45 & 0.32 & 0.74 \\
\hline Social interactions & $4.57(1.61)$ & $6.14(1.09)$ & $4.74(1.47)$ & $5.84(1.24)$ & -0.24 & -0.58 & 0.09 & 0.16 \\
\hline FOSQ & $15.52(3.21)$ & $18.01(2.97)$ & $15.21(3.41)$ & $16.90(3.94)$ & -0.89 & -1.70 & -0.08 & 0.031 \\
\hline ESS & $10.81(4.82)$ & $5.89(3.51)$ & $10.55(4.62)$ & $6.52(4.14)$ & 0.78 & -0.40 & 1.95 & 0.19 \\
\hline BMI $\left(\mathrm{kg} / \mathrm{m}^{2}\right)$ & 33.55 (8.33) & $32.96(8.19)$ & $32.82(7.32)$ & $32.38(7.37)$ & 0.23 & -0.53 & 0.99 & 0.55 \\
\hline
\end{tabular}

The non-inferiority analyses were based on the PP sample according to International Committee for Harmonization (ICH) E9 guidelines. ${ }^{25}$ Non-inferiority of the telemedicine group compared with control was assessed by a one-sided $97.5 \%$ CI for the point estimate of the difference between the two groups, calculated by adequacy of CPAP adherence, using two-sample t tests.

Overall efficacy analyses were conducted using the ITT sample. We evaluated treatment effects by two-sided tests with a significance level of $\alpha=0.05$. Multiple linear regression analysis was performed to assess the relationship between potential predictive factors and CPAP compliance (dependent variable). Details about variable selection are provided in online supplementary material. We compared continuous efficacy measures between groups using analysis of covariance (ANCOVA) models with adjustment for baseline variables and centre. We analysed categorical efficacy and safety measures by using logistic regression models.

All analyses were performed with IBM SPSS Statistics V.20.0.

\section{Cost-effectiveness analysis}

The cost-effectiveness analysis was carried out in a Bayesian framework. ${ }^{26}{ }^{27}$ Considering the asymmetry in the cost distribution and the correlation between effectiveness and cost, we assumed a multivariate normal distribution for the effectiveness and the log-transformed costs. In a Bayesian analysis, the prior distributions for the parameters of the model should be defined. We used non-informative priors to let the sample data control the posterior distribution. We estimated posterior distributions using Markov Chain Monte Carlo methods. ${ }^{28}$ The expected mean effectiveness and costs, and 95\% Bayesian credible interval were then estimated from the posterior distributions. Moreover, we assessed the incremental effectiveness and cost, and the probability that telemedicine would be more effective or cheaper than control. To illustrate the results, we used the cost-effectiveness plane, where the joint posterior distribution of the incremental effectiveness and costs are displayed in an $\mathrm{x}-\mathrm{y}$ plot, and the costeffectiveness acceptability curve (CEAC), where the probability of preference for telemedicine is displayed as a function of the willingness to pay for a QALY. ${ }^{29}$ Furthermore, for certain combinations between groups, we assessed the incremental effectiveness, incremental cost and incremental net benefit.

\section{RESULTS}

Of the 240 patients screened, 139 were randomised and 123 $(88 \%)$ completed the study (figure 1$)$. Data are reported on an

Table 3 Comparison of typical OSA symptoms between the telemedicine and control groups

\begin{tabular}{|c|c|c|c|c|c|c|c|c|}
\hline & \multicolumn{2}{|l|}{ Control } & \multicolumn{2}{|l|}{ Telemedicine } & \multirow{2}{*}{$\begin{array}{l}\text { OR* } \\
\text { (control group data } \\
\text { were reference values) }\end{array}$} & \multicolumn{2}{|c|}{$\begin{array}{l}95 \% \mathrm{Cl} \text { for the } \\
\text { OR }\end{array}$} & \multirow[b]{2}{*}{ p Value } \\
\hline & $\begin{array}{l}\text { Baseline } \\
\mathrm{N}(\%)(\mathrm{N}=70)\end{array}$ & $\begin{array}{l}\text { Follow-up } \\
N(\%)(N=64)\end{array}$ & $\begin{array}{l}\text { Baseline } \\
\mathrm{N}(\%)(\mathrm{N}=69)\end{array}$ & $\begin{array}{l}\text { Follow-up } \\
N(\%)(N=64)\end{array}$ & & Lower & Upper & \\
\hline Snoring & $65(96)$ & $12(19)$ & $68(100)$ & $14(22)$ & 1.13 & 0.46 & 2.78 & 0.78 \\
\hline Witnessed apnoeas & $62(97)$ & 12 (19) & $56(88)$ & $12(19)$ & 1.08 & 0.43 & 2.71 & 0.88 \\
\hline Daytime fatigue & $48(69)$ & $14(22)$ & $49(71)$ & $21(33)$ & 1.85 & 0.82 & 4.20 & 0.14 \\
\hline Nocturia & $39(56)$ & $17(27)$ & $44(64)$ & $15(23)$ & 0.74 & 0.31 & 1.74 & 0.48 \\
\hline Drowsy driving & $42(60)$ & $15(23)$ & $39(57)$ & $12(19)$ & 0.82 & 0.33 & 2.01 & 0.66 \\
\hline
\end{tabular}


Table 4 Cost analysis

\begin{tabular}{|c|c|c|c|c|c|c|}
\hline & \multicolumn{3}{|l|}{ Time or use* } & \multicolumn{3}{|l|}{ Cost } \\
\hline & Control & Telemedicine & p Valuet & Control & Telemedicine & p Valuet \\
\hline Follow-up visit time & $37.53(9.64)$ & 38.97 (12.04) & 0.686 & $35.59(9.15)$ & $35.58(10.20)$ & 0.840 \\
\hline Mask changes & $32(39.7 \%)$ & $19(30 \%)$ & 0.158 & $25.40(34.63)$ & $15.83(25.20)$ & 0.158 \\
\hline Extra visits (physicians) & $7(7.9 \%)$ & $11(16.7 \%)$ & 0.149 & $16.44(65.71)$ & $27.13(63.86)$ & 0.149 \\
\hline Extra visits (nurses) & $10(9.5 \%)$ & $24(28.3 \%)$ & 0.010 & $0.66(2.27)$ & $1.48(2.98)$ & 0.012 \\
\hline GP visits & $28(23.8 \%)$ & $40(35 \%)$ & 0.261 & $20.83(48.22)$ & $27.33(52.12)$ & 0.215 \\
\hline Emergency visits & $3(3.2 \%)$ & $6(6.7 \%)$ & 0.378 & $5.32(31.30)$ & $11.18(44.63)$ & 0.371 \\
\hline Medications added & $16(25.4 \%)$ & $19(31.7 \%)$ & 0.443 & $9.12(33.01)$ & $4.70(14.27)$ & 0.604 \\
\hline Travelling time to the hospital & $23.30(16.48)$ & $8.64(8.42)$ & $<0.001$ & $15.26(10.11)$ & $7.02(7.05)$ & $<0.001$ \\
\hline Time out of work & $55.03(53.05)$ & $21.12(21.21)$ & $<0.001$ & $47.00(48.19)$ & $34.30(35.42)$ & 0.240 \\
\hline
\end{tabular}

ITT basis unless otherwise stated. Both groups had similar baseline characteristics (table 1), except for lower mean age in the control group.

\section{CPAP adherence}

Mean CPAP use was $4.2 \pm 2.0 \mathrm{~h} /$ day in the control group and $4.4 \pm 2.0 \mathrm{~h} /$ day in the telemedicine group $(p=0.827)$. When using the accepted definition of adequate adherence ( $>4 \mathrm{~h} /$ day), $57 \%$ of controls had adequate compliance compared with $65 \%$ of the telemedicine group $(\mathrm{p}=0.329)$.

On bivariate analysis, several variables were significantly associated with CPAP compliance (see online supplementary table $\mathrm{S} 1$ ). Among these variables, in the multivariate analysis CPAP compliance was significantly influenced by age and self-reported daytime fatigue at the end of the study.

In the non-inferiority analysis, using the PP sample the point estimate of the difference in CPAP compliance (telemedicine minus control) was 0.04 . The lower bound of the one-sided $97.5 \%$ CI for the point estimate was -0.72 for the PP sample, which exceeded the previously specified -1 margin and met the criteria for non-inferiority (see online supplementary figure S1).

\section{Clinical outcomes}

As shown in table 2, we found general improvements in QoL and sleepiness (ESS) after 6 months of CPAP treatment, but there were no significant differences in change from baseline between the telemedicine and control groups, except for FOSQ $(-0.89 ; 95 \%$ CI -1.70 to $-0.08 ; \mathrm{p}=0.031)$. There was no change in body mass index (BMI) in either group. Similar reductions were seen in the most common OSA symptoms (snoring, witnessed apnoeas, nocturia) in both groups after CPAP treatment (table 3). The side effects of CPAP treatment were comparable between the two groups at the end of the study (see online supplementary table S2). The two groups reported similar degrees of satisfaction with the follow-up procedures (see online supplementary tables S3 and S4).

\section{Resource use and cost analysis}

Table 4 shows the average use of resources and costs of both follow-up strategies. There were no significant differences in the average lengths of follow-up visits or mask changes. The telemedicine group required more extra visits: $16.7 \%$ and $28.3 \%$ of these patients visited a physician or a nurse, respectively, during the 6-month treatment period, compared to rates of $7.9 \%$ and $9.5 \%$ in the control group, with a significant difference found for nurse visits. Visits to general practitioners and emergency services were more frequent in the telemedicine group, although the differences were not statistically significant. The number of visits to general practitioners was lower when only OSA-related visits were considered (falling from 28 to 12 for the control group and from 40 to 9 for the telemedicine group). Only one visit to the emergency service was OSA related. The main differences between groups concerned the time required for travelling to the hospital and the missed work due to face-to-face visits.

\section{Cost-effectiveness analysis}

Table 5 summarises the cost-effectiveness analysis. The mean total costs were $€ 180.4$ and $€ 168.4$ for the control and telemedicine

Table 5 Cost-effectiveness analysis

\begin{tabular}{|c|c|c|c|c|c|c|}
\hline & \multicolumn{2}{|l|}{ Control } & \multicolumn{2}{|l|}{ Telemedicine } & \multicolumn{2}{|l|}{ Difference } \\
\hline & Mean (SD) & $95 \% \mathrm{Cl}$ & Mean (SD) & $95 \% \mathrm{Cl}$ & Mean (SD) & $95 \% \mathrm{Cl}$ \\
\hline Cost $(€)$ & $180.4(17.01)$ & (151.3 to 218.0$)$ & $168.4(19.65)$ & (135.6 to 212.8 ) & $-11.99(25.98)$ & $(-62.33$ to 40.99$)$ \\
\hline SAHS-related cost $(€)$ & $150.9(13.14)$ & (128.1 to 180.1 ) & $114.5(12.08)$ & (94.13 to 141.5 ) & $-36.35(17.87)$ & $(-36.15$ to -1.35$)$ \\
\hline \multicolumn{7}{|l|}{ Effectiveness } \\
\hline QALYs & $0.0120(0.0169)$ & $(-0.0211$ to 0.0451$)$ & $0.0108(0.0182)$ & $(-0.0250$ to 0.0467$)$ & $-0.0012(0.0248)$ & $(-0.0500$ to 0.0474$)$ \\
\hline Epworth & $5.079(0.5323)$ & (4.034 to 6.124) & $3.932(0.5381)$ & (2.869 to 4.992 ) & $-1.147(0.7552)$ & $(-2.643$ to 0.3317$)$ \\
\hline CPAP use (h) & $4.179(0.2752)$ & (3.633 to 4.717 ) & $4.416(0.2583)$ & (3.911 to 4.922 ) & $0.2377(0.377)$ & $(-0.5009$ to 0.9699$)$ \\
\hline
\end{tabular}


Figure 2 Cost-effectiveness acceptability plane. A scatterplot of the posterior cost and effectiveness differences. Grey points represent OSA-related costs and black points represent total costs. OSA, obstructive sleep apnoea; SAHS, sleep apnoea-hypopnoea syndrome.

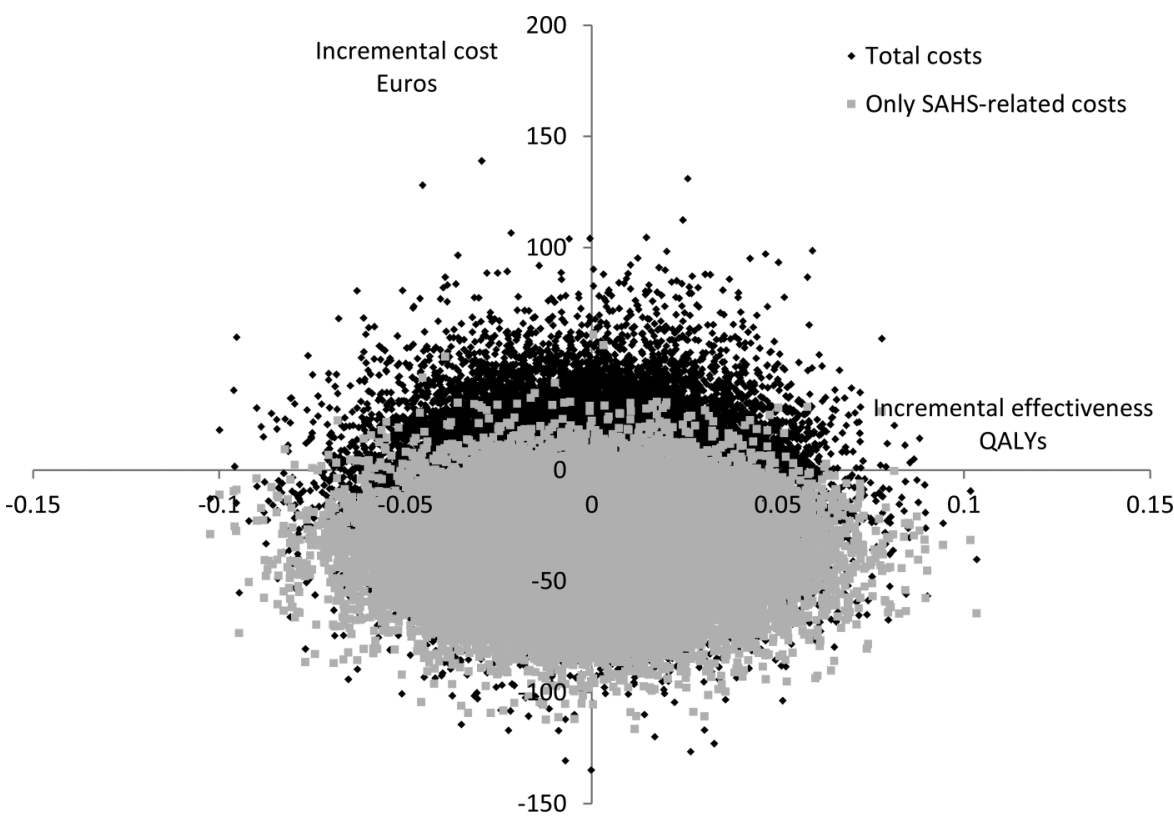

groups, respectively. The estimated probability that telemedicine would be cheaper than control was $68.8 \%$. When only OSA-related visits and drugs were considered, the estimated cost for the control group was $€ 150.9$ and $€ 114.5$ for the telemedicine group; this also increased the probability to $97.9 \%$. The efficacy measure (QALYs) was lower on average for the telemedicine group compared with the control group, although the difference was not statistically significant. The posterior distribution of the incremental effectiveness and incremental cost are showed in figure 2. Figure 3 shows the CEAC. Since the telemedicine-based strategy was cheaper than the hospital-based follow-up, it was preferable in situations with low willingness to pay. At a standard willingness-to-pay threshold of $€ 20000-30000$ per QALY, the two procedures were equally cost-effective. CPAP compliance and ESS improvements were considered alternative measures of effectiveness, but neither resulted in statistically relevant differences.

\section{DISCUSSION}

In this multicentre RCT, we compared a new telemedicine-based follow-up strategy for OSA against standard face-to-face follow-up over a 6-month treatment period. Although the study groups achieved similar levels of CPAP use and clinical outcomes, probably due to the low possibility of further improvement in our setting, where levels of compliance are generally high, ${ }^{30}$ analyses showed telemedicine to be more costeffective, with travel costs and lost work time being the most important sources of savings. Interestingly, the telemedicine group made more extra visits than the face-to-face group, but most of them were non-OSA-related. This may be explained by the fact that telemedicine improved the communication between professionals and patients, thereby increasing the detection of non-OSA-related problems.

CPAP is the optimal treatment for OSA, ${ }^{31}$ but adherence fails in some subjects. After analysing the relationship between compliance and effects on symptoms in 30 articles (2047 participants), a 2014 Cochrane review emphasised the need for new strategies that promote CPAP compliance and assessment of their cost-effectiveness. ${ }^{32}$ Our study analysed these two aspects (adherence to treatment and cost-effectiveness) in a novel telemedicine-based approach for CPAP follow-up.

The application of ICT is increasing in clinical practice for different diseases but prudence is required, especially after some recent negative results. ${ }^{12} 13$ Some authors have highlighted the need for a better understanding of the factors that predict
Figure 3 Cost-effectiveness acceptability curve showing the cost-effectiveness probabilities for telemedicine-based follow-up by different degrees of willingness to pay for QALYs. QALY, quality-adjusted life year; SAHS, sleep apnoea-hypopnoea syndrome.

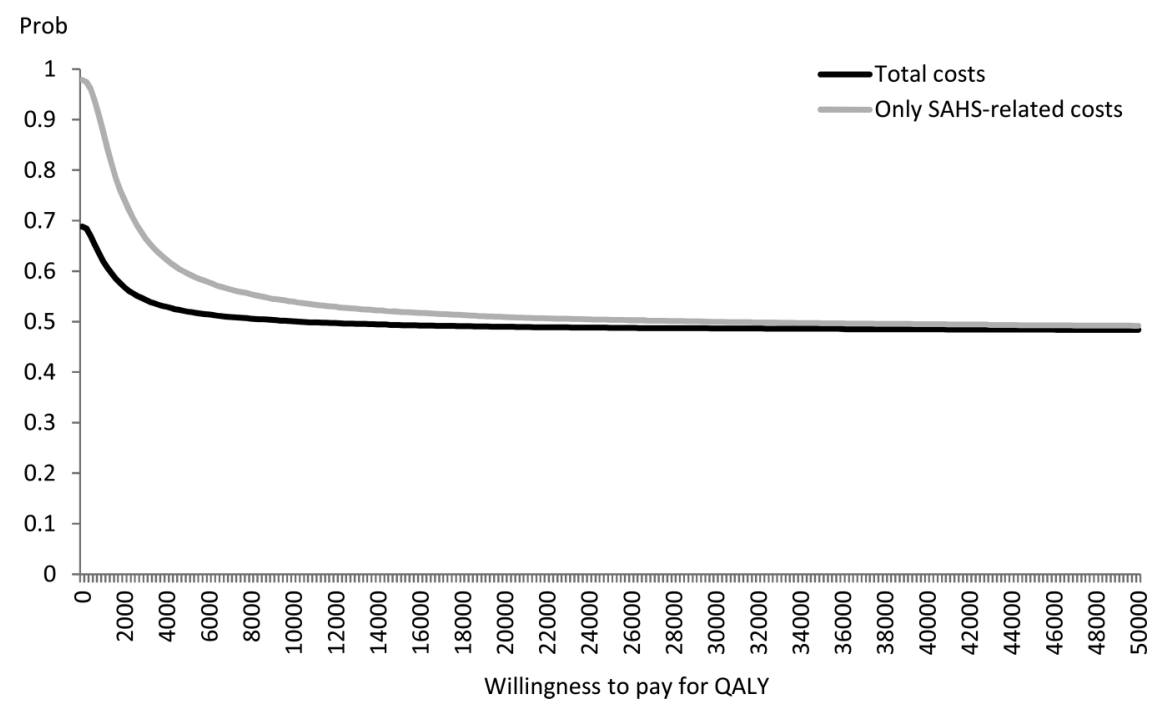


success in telemedicine programmes before they are widely implemented, stressing the importance of appropriate patient selection. ${ }^{33}$ In a previous study we demonstrated that an OSA population could benefit from a telemedicine-based management approach. ${ }^{34}$

Diverse results have been found for telemedicine interventions designed to improve CPAP compliance in OSA. ${ }^{14-18}$ Sparrow et $a l^{14}$ randomly assigned 250 patients starting CPAP therapy either to a telephone-linked interactive voice-response system or to an attention placebo control for 12 months. Patients in the telemedicine group showed improvements but result interpretation is limited due to generally low CPAP adherence. ${ }^{14}$ Similar limitations were found in another RCT that assessed the application of a CPAP wireless telemonitoring system. ${ }^{15}$ Other telemedicine interventions, such as televisits, improved CPAP adherence in a small group of non-compliant OSA patients. ${ }^{16}$ Nevertheless, other studies have not found any positive effects. ${ }^{17} 18$ In summary, the results vary widely, and most studies were single-centre with low levels of CPAP compliance and small sample sizes. Moreover, very few performed cost-effectiveness analyses.

In a recent systematic review of the cost-effectiveness of telemedicine, the authors stated that, even though the use of this technological approach has grown over the last 30 years, decisions are still influenced by financial constraints. ${ }^{35}$ As mentioned, Wilson and colleagues ${ }^{33}$ reached similar conclusions. Therefore, the cost-benefit ratio of telemedicine-based health strategies has still to be established. The Bayesian costeffectiveness analysis used in this paper is well suited to this scenario, where there are small differences in the effectiveness of the proposed strategies; that is, where there is a nonnegligible probability of a very small value for the denominator of the incremental cost-effectiveness ratio. In that situation, the Bayesian model allows us to estimate the parameters of interest by introducing a non-informative prior density.

In our analyses we identified as key factors the costs related to patients' transport to the hospital and missed work time. Therefore, our telemedicine-based approach could be especially advantageous if applied to the working population and to residents in medically underserved areas. It is also reasonable to expect that this new management strategy could be suitable in clinical settings or health systems that achieve lower compliance levels with conventional follow-up. As it should be for any treatment option, appropriate patient selection remains a key issue. Also, specific training of the staff involved in long-distance follow-up would be necessary for optimal implementation.

As recommended by major guidelines regarding the application of cost-effectiveness analysis, we adopted the societal perspective where all costs are considered regardless of who pays. However, from the healthcare payer perspective, the telemedicine-based strategy would be more expensive than face-to-face follow-up (mean total costs would be $€ 126.6$ and $€ 116.8$, respectively), confirming that the main savings are related to patients' transport to hospital and loss of productivity.

Although the economic analyses in this study were rigorous and extensive, some limitations are worthy of note. We did not consider the indirect hospital costs or the costs of the equipment needed to connect to the website. In addition, it could be argued that a significant number of patients with OSA do not have sufficient computer expertise; in our study approximately $40 \%$ of screened patients lacked such skills. However, given the current exponential growth of internet and mobile phone use, this percentage is likely to decrease over the next few years.
In this multicentre RCT we compared standard hospital-based CPAP follow-up with a novel telemedicine-based strategy. We conclude that the telemedicine procedure was more costeffective due to savings related to transport and productivity losses. Patient satisfaction and side effects were similar. Therefore, telemedicine represents a cost-effective strategy to support the routine clinical follow-up of OSA patients receiving CPAP treatment. Moreover, we believe that a telemedicine-based strategy could provide an excellent alternative approach when adequate face-to-face consultations are impractical.

\section{Author affiliations}

${ }^{1}$ Unitat de Biofísica i Bioenginyeria, Facultat de Medicina, Universitat de Barcelona, Barcelona, Spain

${ }^{2}$ Institut d'Investigacions Biomèdiques August Pi i Sunyer (IDIBAPS), Barcelona, Spain

${ }^{3}$ Centro de Investigación Biomédica en Red de Enfermedades Respiratorias

(CIBERES), Madrid, Spain

${ }^{4}$ Departamento de Métodos Cuantitativos y TiDES Institute, Universidad de Las Palmas de Gran Canaria, Las Palmas de Gran Canaria, Islas Canarias, Spain

${ }^{5}$ Unidad del Sueño, Servicio de Neumología, Hospital Universitario de Bellvitge,

IDIBELL, Hospitalet de Llobregat, Barcelona, Spain

${ }^{6}$ Servicio de Neumología, Hospital San Pedro de Alcántara, Cáceres, Spain

${ }^{7}$ Unidad del Sueño, Hospital Universitario Reina Sofía, Universidad de Córdoba,

IMIBIC, Córdoba, Spain

${ }^{8}$ Unidad Funcional de Sueño, Hospital Universitario Araba, Vitoria-Gasteiz, Spain

${ }^{9}$ Servicio de Neumología, Hospital Universitario de Valme, Sevilla, Spain

${ }^{10}$ Servicio de Neumología, Unidad del Sueño, Hospital Clínic de Barcelona,

Universidad de Barcelona, Barcelona, Spain

${ }^{11}$ Servicio de Neumología, Hospital Germans Trias i Pujol, Universidad Autónoma de

Barcelona, Badalona, Spain

${ }^{12}$ Servicio de Neumología, Hospital Universitario Sagrat Cor, Universidad de

Barcelona, Barcelona, Spain

${ }^{13}$ Servicio de Neumología, Unidad del Sueño, Hospital Universitario Vall d'Hebron,

Barcelona, Spain

${ }^{14}$ Servicio de Neumología, Hospital Universitario Son Espases, IdisPa, Palma de Mallorca, Spain

${ }^{15}$ Departamento de Neurofisiología Clínica y Unidad del Sueño, Hospital Universitario La Ribera, Alzira, Valencia, Spain

${ }^{16}$ Departamento de Fisiología, Universidad de Valencia, Valencia, Spain

Acknowledgements The authors would thank Mr. Albert Gabarrus for his support with statistical analysis of the study data. A special thanks to Esteve-Teijin for their support.

Collaborators Spanish Sleep Network: Ester López, Hospital Universitario de Bellvitge, Hospitalet del Llobregat, Spain; Mercè Gasa, Hospital Universitario de Bellvitge, Hospitalet del Llobregat, Spain; Estefania García-Ledesma, Hospital San Pedro de Alcántara, Cáceres, Spain; Maria-Isabel Rosco-Due, Hospital San Pedro de Alcántara, Cáceres, Spain; Joaquín Durán, Unidad del Sueño, Hospital Universitario Araba, Vitoria-Gasteiz, Spain; Nuria Reyes-Nuñez, Hospital Universitario de Valme, Sevilla, Spain; Susana Pou, Hospital Germans Trias i Pujol, Universidad Autónoma de Barcelona, Badalona, Spain; Félix del Campo, Servicio de Neumología, Universidad de Valladolid, Valladolid, Spain; Gabriel Sampol, Hospital Universitario Vall d'Hebron, Barcelona, Spain; Odile Romero, Hospital Universitario Vall d'Hebron, Barcelona, Spain; Marta Torres, Centro de Investigación Biomédica en Red de Enfermedades Respiratorias (CIBERES), Madrid, Spain.

Contributors All the authors are responsible for and confirm the accuracy and completeness of the data and analyses. $\mathrm{VI}$ and JMM are the guarantors of the manuscript. VI was the clinical research fellow for the trial, participated in the trial design, was responsible for creating the website and for the oversight of the trial, and is the first author of the report. MAN, FJVP and MME were responsible for the cost-effectiveness analyses and for the revision of the final version of the manuscript. CM, JFM, NF, AA, FCR, CR, JA, MG, PLL, CE, MP, JP, MD, NS, JC, BJ, $C L, C E, A M, O P, M A$ and $R C$ contributed to the study concept, data collection and approval of the final version of the manuscript. VI, CE, MD, RF and JMM contributed to the data interpretation and manuscript preparation. FJVP, RF and JMM contributed to the study concept and design, and provided critical revision of the manuscript.

Funding This project was supported by SEPAR/FIS PI14/00416 and ECO2013-47092 (MINECO, Spain).

\section{Competing interests None declared.}

Ethics approval The Ethics Committee of each of the participating centres approved this study.

Provenance and peer review Not commissioned; externally peer reviewed. 


\section{REFERENCES}

1 Peppard PE, Young T, Barnet JH, et al. Increased prevalence of sleep-disordered breathing in adults. Am J Epidemiol 2013;177:1006-14.

2 Dempsey JA, Veasey SC, Morgan BJ, et al. Pathophysiology of sleep apnea. Physiol Rev 2010;90:47-112.

3 Rotenberg B, George C, Sullivan K, et al. Wait times for sleep apnea care in Ontario: a multidisciplinary assessment. Can Respir J 2010;17:170-4.

4 Sánchez de la Torre M, Campos-Rodriguez F, Barbé F. Obstructive sleep apnoea and cardiovascular disease. Lancet Respir Med 2013;1:61-72.

5 Wozniak DR, Lasserson TJ, Smith I. Educational, supportive and behavioral interventions to improve usage of continuous positive airway pressure machines in adults with obstructive sleep apnoea. Cochrane Database Syst Rev 2014;1:CD007736.

6 Sawyer AM, Gooneratne NS, Marcus CL, et al. A systematic review of CPAP adherence across age groups: clinical and empiric insights for developing CPAP adherence interventions. Sleep Med Rev 2011;15:343-56.

7 Chai-Coetzer CL, Luo YM, Antic NA, et al. Predictors of long-term adherence to continuous positive airway pressure therapy in patients with obstructive sleep apnea and cardiovascular disease in the SAVE study. Sleep 2013;36:1929-37.

8 Weaver TE, Grunstein RR. Adherence to continuous positive airway pressure therapy: the challenge to effective treatment. Proc Am Thorac Soc 2008;5:173-8.

9 Schwab RJ, Badr SM, Epstein LJ, et al. ATS Subcommittee on CPAP Adherence Tracking Systems. An official American Thoracic Society statement: continuous positive airway pressure adherence tracking systems. The optimal monitoring strategies and outcome measures in adults. Am J Respir Crit Care Med 2013;188:613-20.

10 Drake C, Day R, Hudgel D, et al. Sleep during titration predicts continuous positive airway pressure compliance. Sleep 2003;26:308-11.

11 Paré $G$, Jaana $M$, Sicotte $C$. Systematic review of home telemonitoring for chronic diseases: the evidence base. J Am Med Inform Assoc 2007;14:269-77.

12 Chaudhry SI, Mattera JA, Curtis JP, et al. Telemonitoring in patients with heart failure. N Engl J Med 2010;363:2301-9.

13 Takahashi PY, Pecina JL, Upatising B, et al. A randomized controlled trial of telemonitoring in older adults with multiple health issues to prevent hospitalizations and emergency department visits. Arch Intern Med 2012;172:773-9.

14 Sparrow D, Aloia M, Demolles DA, et al. A telemedicine intervention to improve adherence to continuous positive airway pressure: a randomised controlled trial. Thorax 2010;65:1061-6.

15 Fox N, Hirsch-Allen AJ, Goodfellow E, et al. The impact of a telemedicine monitoring system on positive airway pressure adherence in patients with obstructive sleep apnea: a randomized controlled trial. Sleep 2012;35:477-81.

16 Smith $C E$, Dauz ER, Clements $F$, et al. Telehealth services to improve nonadherence: a placebo-controlled study. Telemed J E Health 2006;12:289-96.

17 Taylor Y, Eliasson A, Andrada T, et al. The role of telemedicine in CPAP compliance for patients with obstructive sleep apnea syndrome. Sleep Breath 2006:10:132-8.
18 Stepnowsky CJ, Palau JJ, Marler MR, et al. Pilot randomized trial of the effect of wireless telemonitoring on compliance and treatment efficacy in obstructive sleep apnea. J Med Internet Res 2007;9:e14

19 Lloberes P, Durán-Cantolla J, Martínez-García MÁ, et al. Diagnóstico y tratamiento del síndrome de apneas-hipopneas del sueño. Diagnosis and treatment of sleep apnea-hypopnea syndrome. Arch Bronconeumol 2011;47:143-56.

20 Masa JF, Jiménez A, Durán J, et al. Alternative methods of titrating continuous positive airway pressure: a large multicenter study. Am J Respir Crit Care Med 2004; 170:1218-24.

21 Armfield NR, Gray LC, Smith AC. Clinical use of Skype: A review of the evidence base. J Telemed Telecare 2012;18:125-7.

22 Williams A. The role of the EuroQol Instrument in QALY calculations. Working Paper 130. University of York, 1995

23 Richardson G, Manca A. Calculation of quality adjusted life years in the published literature: a review of methodology and transparency. Health Econ 2004;13:1203-10.

24 Badia X, Roset M, Montserrat S, et al. The Spanish version of EuroQol: a description and its applications. Med Clin (Barc) 1999;112:79-85.

25 ICH harmonised tripartite guideline-E9 Statistical principles for clinical trials. 5 February 1998. http://www.ich.org/fileadmin/Public_Web_Site/ICH_Products/ Guidelines/Efficacy/E9/Step4/E9_Guideline.pdf (accessed 23 Jun 2015).

26 Briggs $\mathrm{AH}$. A Bayesian approach to stochastic cost-effectiveness analysis. Health Econ 1999;8:257-61.

27 O'Hagan A, Stevens JW, Montmartin J. Bayesian cost-effectiveness analysis from clinical trial data. Stat Med 2001;20:733-53.

28 Gilks WR, Richardson S, Spiegelhalter DJ. Markov Chain Monte Carlo in Practice. London: Chapman \& Hall/CRC, 2000.

29 Fenwick E, O'Brien BJ, Briggs A. Cost-effectiveness acceptability curves-facts, fallacies and frequently asked questions. Health Econ 2004;13:405-15.

30 Barbé F, Durán-Cantolla J, Capote F, et al. Long-term effect of continuous positive airway pressure in hypertensive patients with sleep apnea. Am J Respir Crit Care Med 2010;181:718-26.

31 Martínez-García MA, Capote F, Campos-Rodríguez F, et al. Effect of CPAP on blood pressure in patients with obstructive sleep apnea and resistant hypertension: the HIPARCO randomized clinical trial. JAMA 2013;310:2407-15.

32 Balk EM, Chung M, Chan JA, et al. Future Research Needs for Treatment of Obstructive Sleep Apnea: Identification of Future Research Needs From Comparative Effectiveness Review No. 32. AHRQ Publication No. 12-EHC033-EF, February 2012.

33 Wilson RS, Cram P. Another sobering result for home telehealth-and where we might go next. Arch Intern Med 2012;172:779-80.

34 Isetta V, León C, Torres $M$, et al. Telemedicine-based approach for obstructive sleep apnea management: building evidence. Interact J Med Res 2014;3:e6.

35 Mistry H. Systematic review of studies of the cost-effectiveness of telemedicine and telecare. Changes in the economic evidence over twenty years. J Telemed Telecare 2012;18:1-6. 